\title{
Completely multimode arrayed waveguide grating-based wavelength demultiplexer
}

\author{
A.A.M. Kok, S. Musa, A. Borreman, M.B.J. Diemeer and A. Driessen \\ Integrated Optical Microsystems, MESA Research Institute, University of Twente \\ P.O. Box 217, 7500 AE Enschede, The Netherlands \\ email: M.B.J.Diemeer@utwente.nl
}

\begin{abstract}
Abstraci-A multimode fiber-matched arrayed waveguide grating-based demultiplexer has been demonstrated. In this design, multimode waveguides have been used as array elements. The device, which can be used in short-distance communications, has been realized using low-cost polymer planar waveguide technology. Three wavelength channels were succesfulty separated. The average fiber-to-fiber insertion loss was $14 \mathrm{~dB}$ and the worst-case cross talk was $<-12.5 \mathrm{~dB}$.

Keywords-Arrayed Waveguide Grating, multimode waveguides, polymer waveguides
\end{abstract}

\section{INTRODUCTION}

$\mathrm{T}$ HE demand for high bitrates in Local Area Networks (LANs) is increasing rapidly. In order to meet these bitrates. optical data transmission is required. LANs are nowadays built with a combination of optical and electrical data tranmission lines. These optical connection lines allow bitrates of about $600 \mathrm{Mbps}$. Since LANs are shared by a relatively low number of users, the costs are more important than they are in long-haul networks. To reduce these costs, cheap receivers, transmitters, fibers and components like splitters and switches are necessary. Transmitters that are used in these optical networks, like Vertical Cavity Surface Emitting Lasers (VCSELs), are operating in the wavelength range from 600 to $1000 \mathrm{~nm}$. In this window, a simple Si-detector can be used as a receiver. Multimode fibers are used since they have relaxed alignment tolerances and a less stringent fabrication precision.

A major drawback of multimode technology is modal dispersion, which limits the transmission capacity of the multimode fiber. One of the solutions to increase the capacity of LANs to $>1 \mathrm{Gbps}$ is Wavelength Division Multiplexing (WDM). Since the 1990s, multimode fiber-matched WDMs are being developed using diffraction gratings or employing dielectric filters in different configurations [1]. A very cost effective WDM based on an Arrayed Waveguide Grating (AWG) used in the singlemode regime has shown excellent performance [2].

In this article the design, fabrication and characterizations of an AWG-based demultiplexer based on completely multimode waveguides are given. The device is fabricated using very low-cost polymer planar waveguide technology. Polymer planar waveguide technology is a cost effective technology for fabrication of large cross-sectional multimode devices since a wide range of cheap optical polymers is commercially available, relatively thick waveguides can be fabricated easily using very simple equipments and it is suitable for mass fabrication especially when techniques such as molding or photo patterning are used [3],[4].

In section II the theory of a multimode AWG-based demul-

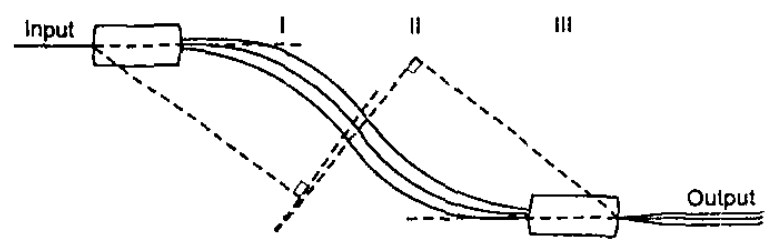

Fig. 1. Layout of a demultiplexer based on an anti-symmetrical or s-bend aray geometry.

tiplexer will be treated. The fabrication process is described in section III. In section IV the design of a completely multimode demultiplexer is given and in section $\mathrm{V}$ the characterization of the device is described. Finally, conclusions are given in section VI

\section{THEORY}

\section{A. Operation principle}

To design an arrayed waveguide grating-based demultiplexer, different array geometries can be applied [2], [5], [6]. A symmetrical waveguide array was first introduced in 1991 by Smit [2] and is now widely used in singlemode optical communication networks. Another array geometry is the antisymmetrical or s-bend layout, which is shown in Fig. II-A. This design was first proposed by Adar et al. in 1993 [6]. In order to properly design and optimize the anti-symmetrical array, it is divided into three sections. Due to the opposite curvatures of sections I and III, the dispersion of the first section is compensated by the last section. Section II accounts for the total dispersion of the array. The path length difference $\Delta L$ between the waveguides in this part of the array equals an integer multiple $m$ (called the order) of the central wavelength in the array:

$$
\Delta L=m \lambda_{c} / N_{e f f, c}
$$

where $\lambda_{c}$ is the central wavelength in vacuum and $N_{c f f, c}$ is the effective refractive index of the array waveguide modes at that wavelength. The choice of array geometry will be explained in this section.

At the central wavelength, the fields at the array output aperture of all channels have the same relative phase (apart from an integer multiple of $2 \pi$ ) and intensity distribution. In this way the phase and intensity distribution at the input aperture of the array is reproduced at the output aperture. The light propagating at the central wavelength $\lambda_{c}$ coming out of the array converges 
EUROCON 2003 Ljubljana, Slovenia

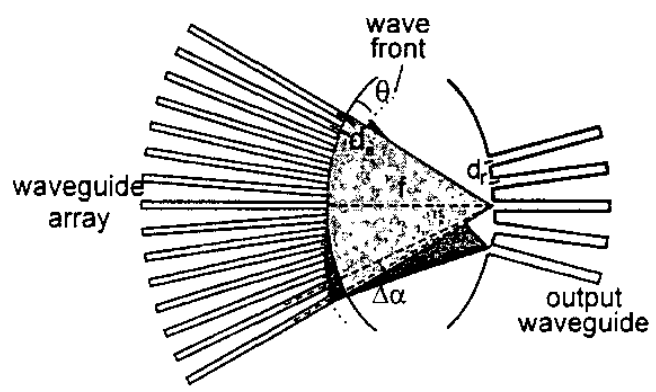

Fig. 2. Layout of the output Free Propagation Region.

in the output FPR and is focused into the central output channel in the image plane.

If the wavelength is shifted to $\lambda_{c}+\Delta \lambda$, there will be a phase change in the individual waveguides that increases linearly from the lower to the upper channel. As a result, the phase front at the output aperture of the array will be slightly tilted, so the beam is focused on a different position in the image plane. This is illustrated in Fig. 2. The tilting angle $\theta_{m}$ of the light propagating at a wavelength $\lambda$ is given by

$$
\theta_{m} \simeq \frac{N_{c f f}}{N_{f}} \frac{\Delta L-m \lambda_{g}}{d_{a}}
$$

where $N_{e f f}$ and $N_{f}$ are the effective refractive indices in the waveguide array and in the FPRs, respectively, $d_{a}$ is the pitch between the array waveguides and $\lambda_{g}=\lambda / N_{\text {eff }}$. By placing output waveguides at specific locations in the image plane, a spatial separation of the wavelength channels is achieved [2].

\section{B. Modal dispersion}

The difference in the effective refractive indices in the FPR and in the array waveguides is in our case very small, so $N_{e f f} / N_{f} \simeq 1$. The spectral dispersion $D_{m}$ is defined as the lateral displacement of the focal spot on the image plane per unit wavelength change. If $s$ denotes the absolute lateral displacement in the image plane and $f$ is the focal length of the array, the dispersion can be expressed as

$$
D_{m}=\frac{d s_{m}}{d \lambda_{g}}=f \frac{d \theta_{m}}{d \lambda_{g}}=-\frac{N_{e f f}}{N_{f}} \frac{m}{\Delta \alpha} \simeq-\frac{m}{\Delta \alpha}
$$

where $\Delta \alpha=d_{a} / f$ is the divergence angle of the array channels in the array aperture.

Besides dispersion due to the wavelength shift, dispersion also occurs if more than one mode propagates at the same wavelength through the array channels. This so-called modal dispersion can be seen by reviewing Eq. (2), which can be rewritten as

$$
\Delta \theta_{m}=\left(\frac{\lambda}{N_{e f f}}-\frac{\lambda_{c}}{N_{e f f, c}}\right) \frac{m}{d_{a}}
$$

In principle, this formula gives the displacement of the focal spot in the image plane of a certain wavelength $\lambda$ with respect to the focal spot of the central wavelength. However, the same formula can be used to calculate the worst-case displacement of the focal spot of a certain wavelength due to modal dispersion. As a worst-case estimation, the effective refractive index contrast between different modes propagating at the same wavelength could be taken equal to the refractive index contrast between the core and cladding materials. Then, the displacement of the focal spot of the central wavelength due to modal dispersion can be determined by

$$
\Delta \theta_{\text {mod.disp. }}=\left(\frac{\lambda_{c}}{n_{\text {cladding }}}-\frac{\lambda_{c}}{n_{\text {core }}}\right) \frac{m}{d_{a}}
$$

The same formula can be used to calculate the modal dispersion of any wavelength channel. To improve the approximation, the effective indices of the fundamental mode and the highest order mode should be substituted in the expression for $\Delta \theta_{\text {mod.disp. }}$. In general, modal dispersion disturbs the exact reproduction of the input field intensity at the image plane, because the focal spot is spread out in one direction. In case the array is multimode, the positions of the receiver waveguides should be optimized to make sure that no light is coupled into an undesired waveguide.

Combining Eqs. (1) and (4) it can be seen that the dispersion increases linearly with increasing path length difference between the array waveguides. This means that if the length difference is kept small, the dispersion will be very small as well, thus broadening the range of wavelengths that are focused on the image plane. In order to design an AWG-based demultiplexer for application in coarse WDM, the anti-symmetrical array waveguide geometry of Adar [6] is applied.

\section{Spectral response}

The field in the image plane at the position of the $k$-th receiver channel is given by [6]

$$
E_{k}(\lambda)=\sum_{j=1}^{n} f_{j}^{2} \exp \left[i j 2 \pi N_{e f f}\left(\Delta L+d_{a} \theta_{k}\right) / \lambda\right]
$$

where $f_{j}$ is determined by the far field of the input and output channels. By good approximation, the field intensity in these channels can be assumed to have a square distribution. From Fourier optics we know that the far field will be a $\sin c^{2}$-function so the coupling coefficient $f_{j}$ can be expressed as

$$
f_{j}=\frac{\sin ^{2}[\pi / n(j-(n+1) / 2)]}{[\pi / n(j-(n+1) / 2)]^{2}}
$$

The intensity at the position of the $k$-th receiver waveguide is given by

$$
I_{k}(\lambda)=\left|E_{k}(\lambda)\right|^{2}
$$

The spectral response of this receiver waveguide follows as

$$
T_{k}(\lambda)=-10 \log \frac{I_{k}\left(\lambda_{k}\right)}{I_{k}(\lambda)}
$$

where the transmission has been normalized so its maximum is $0 \mathrm{~dB}$.

Fig. 3 shows the spectral transmission of one of the receiver waveguides of an AWG of order 3 with 17 array waveguides. The receiver waveguide was designed for a wavelength of $850 \mathrm{~nm}$. In the figure the different orders that are coupled into this output waveguide are shown. With increasing order $m$ (i.e. 


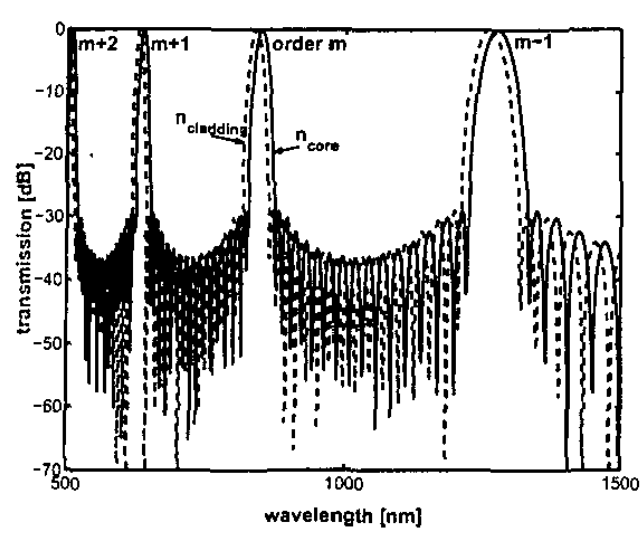

Fig. 3. Spectral response of the lowest and highest order mode at the same wavelength $850 \mathrm{~nm}$ of an AWG with $m=3$ and $n=17$.

increasing optical path length difference $\Delta L$ ), the peaks become narrower and the Free Spectral Range (FSR) decreases.

The FSR can be found by comparing the optical path length difference of orders $m-1$ and $m$

$$
\Delta L=m \frac{\lambda_{0}}{N_{e f f, 0}}=(m-1) \frac{\lambda_{0}+\Delta \lambda_{F S R}}{N_{e f f}}
$$

Let us define an effective index difference as

$$
\Delta N=N_{e f f, 0}-N_{e f f}
$$

After some manipulation, the FSR follows from Eq. (10) as

$$
\Delta \lambda_{F S R}=\lambda_{0} \frac{1-m \Delta N / N_{e f f, 0}}{m-1}
$$

If we compare the optical path length difference of the $m$-th order to that of the $(m+1)$-th order, we find the FSR to be

$$
\Delta \lambda_{F S R}=\lambda_{0} \frac{1+m \Delta N / N_{e f f, 0}}{m+1}
$$

In Fig. 3, the transmission of another mode propagating at the same wavelength is also depicted. The two modes for which the transmission spectrum is shown are the highest and lowest order mode, where the effective refractive index contrast is taken to be equal to the contrast between the indices of core and cladding material, as indicated in the figure. In a high-order narrowband AWG, where the spacing $\Delta \lambda$ between the wavelengths is small, broadening due to modal dispersion might cause successive spectral channels to overlap, thus degrading device performance. The lower the order of the AWG, the larger the FSR will be. In that case the spectral channels can still be separated even if the channel spacing is large.

\section{FABRICATION PROCESS}

The device is made on a $100 \times 100 \times 1 \mathrm{~mm}^{3}$ borosilicate glass substrate with a refractive index of 1.47 at $850 \mathrm{~nm}$ wavelength. As a core material, a polymer called SU-8 is used, which is a negative photoresist [7]. It is highly transparent for wavelengths
$>800 \mathrm{~nm}$, has shown heat resistance to temperatures $>200$ degrees Celsius and it is chemically and mechanicaliy stable [8]. The index of SU-8 at a wavelength of $850 \mathrm{~nm}$ is 1.58 .

The SU-8 is spin-coated on the glass substrate. By varying the spin speed, the height of the layer can be varied from 10 to $60 \mu \mathrm{m}$. Between two waveguides of $40 \mu \mathrm{m}$ high, the smallest gap that can be made is $4 \mu \mathrm{m}$ wide. The layer is pre-baked at 95 degrees Celsius to evaporate the solvent. The film is exposed to UV-light with a mask. Following the UV-exposure, the film is post-baked at a temperature of again 95 degrees Celsius. In this post exposure baking step the cross-linking of the polymer takes place in all exposed areas. The SU-8 film is developed in RER 600 (PGMEA). Finally, the defined structures are exposed to UV-light and hard-baked at 150 degrees Celsius [8].

The cladding is a thermally cross-linked copolymer from HEMA and Styrene with an index of 1.554 at $850 \mathrm{~nm}$. The refractive indices of the materials were measured using an Abbe refractometer [9].

\section{DESIGN}

The fiber-chip losses of the demultiplexer are reduced by adjusting the Numerical Aperture (NA) and the waveguide dimensions of the access waveguides to that of the multimode fibers. The NA is defined as

$$
N A=\sqrt{n_{\text {core }}^{2}-n_{\text {cladding }}^{2}}
$$

The SU-8 waveguides thus have a NA of 0.29 .

To couple light in and out of the device, $50 / 125 \mu \mathrm{m}$ graded index multimode fibers with a NA of 0.2 were used. To reduce the fiber-chip losses, the dimensions of input and output access waveguides are taken to be $40 \times 40 \mu \mathrm{m}^{2}$. Cut-back measurements have been carried out to experimentally determine the waveguide properties. By changing the stress in the input multimode fiber, different sets of modes can be generated. With these different sets of modes, the mode and polarization dependent loss can be determined. The fiber-chip losses of a $40 \times 40 \mathrm{\mu m}^{2}$ SU-8 waveguide are $\sim 1.3 \mathrm{~dB}$ worst-case of the two facets in total. The worst-case propagation loss of such a waveguide is $\sim 0.5 \mathrm{~dB} / \mathrm{cm}$.

An important building block of the AWG-based demultiplexer are the bends, especially in the array waveguides. In bent waveguides, two contributions to bend losses need to be taken in to account [10]. At first, light is lost due to radiation of light as it propagates along the bend of constant radius. The second contribution to bend losses occurs at the transition from the straight waveguide to the bend due to modal mismatch. This adds an offset to the total bend losses. The losses occurring at the junctions between straight waveguides and bends, or between bends of different radii, are expected to be more dominant when the number of modes in the waveguide increases [11].

To keep the number of modes in the array waveguides low, they are are designed to be $10 \mu \mathrm{m}$ wide. Experiments have shown that the bend losses of a $10 \times 40 \mu \mathrm{m}^{2}$ SU-8 waveguide are $<0.4 \mathrm{~dB}$ down to a bending radius of $5 \mathrm{~mm}$. The gaps between the array waveguides at the array apertures are $4 \mu \mathrm{m}$, which is the smallest gap that can be reproducibly processed.

The $1 \times 3$ AWG-based demultiplexer that is designed has a central wavelength of $850 \mathrm{~nm}$ and a spectral channel spacing of 


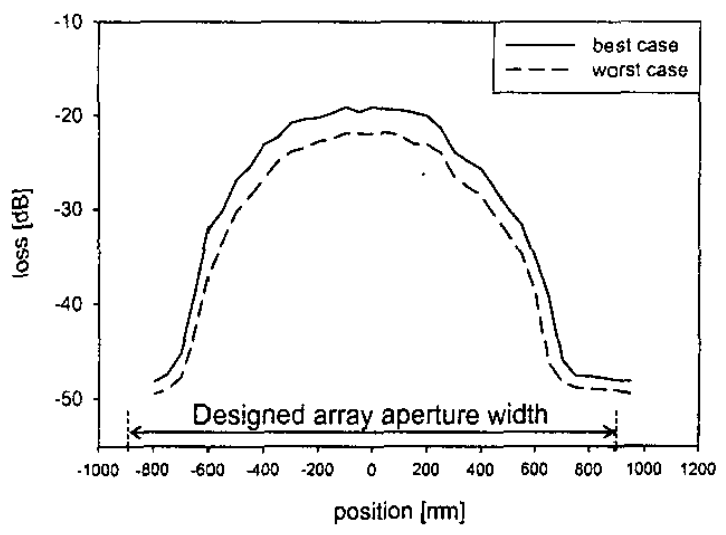

Fig. 4. The best-case and worst-casc intensity distributions at the input aperture of the array. The length of free propagation was $4.9 \mathrm{~mm}$. The far fi eld width from a calculation based on the NA of the input aperture is also shown.

$50 \mathrm{~nm}$. The waveguide array is of order $m=5$. The focal length of the AWG is $4.9 \mathrm{~mm}$. The pitch of the output waveguides is designed to be $55 \mu \mathrm{m}$, so the gap between them equals $15 \mu \mathrm{m}$. This distance should be enough to avoid cross talk due to modal dispersion.

In order to design the number of array waveguides, the far field width of the input waveguide has been measured as well as calculated. This calculation, based on the NA of the input waveguide, predicted a far field width of $\sim 1.8 \mathrm{~mm}$. The far field was experimentally determined by scanning with a multimode fiber along the plane of the array input aperture. The results are shown in figure 4 . In this design, the array consists of 127 waveguides, resulting in an array aperture width of $1780 \mu \mathrm{m}$ (see figure 4).

As soon as section I of the array (see figure II-A) is designed, the design parameters for section III are also determined, since these sections are identical (apart from a rotation). Sections I and III consist of a set of array waveguides that increase linearly in length. Each waveguide is composed of two straight waveguides with a curved part in between and reaches the intersection line under a normal angle. At the intersection line the array waveguides are equally spaced.

The length difference $\Delta L$ between adjacent array waveguides in section 1 can be chosen arbitrarily, but it is an important parameter in the scaling of the device. In the present design it is chosen to be $60 \mu \mathrm{m}$. The optimum bending angle of the central array waveguide is found to be 51.0 degrees $(0.89$ radian). The size of the demultiplexer is $55 \times 21 \mathrm{~mm}^{2}$.

\section{RESULTS}

For measuring the transmission of the fabricated AWG-based demultiplexers, a halogen lamp with a broad spectrum was used as light source. Multimode $50 / 125 \mu \mathrm{m}$ graded-index fibers were used to couple light in and out of the device. The transmission was measured using a spectrometer (Spectro 320 of Instrument Systems) with a resolution of $5 \mathrm{~nm}$.

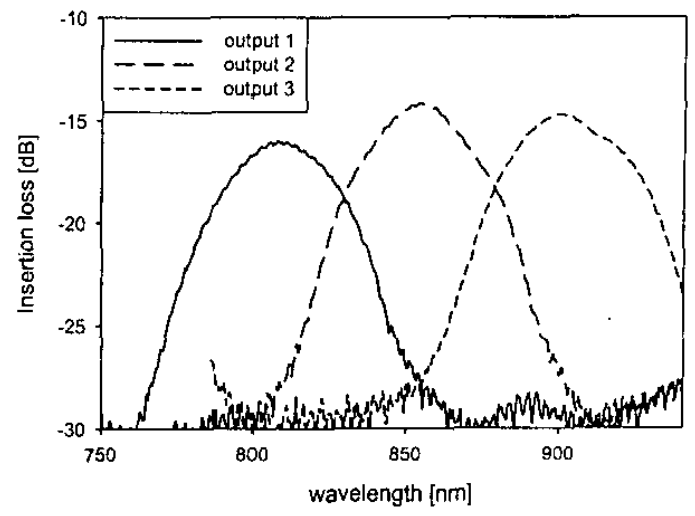

Fig. 5. Measured insertion loss spectrum of an anti-symmetrical demultiplexer with $\lambda_{c}=850 \mathrm{~nm}, \Delta \lambda=50 \mathrm{~nm}(\mathrm{~m}=5, n=127)$. Shown are the three wavelength peaks for which the demultiplexer was designed.

Fig. 5 shows the insertion loss spectrum of the $1 \times 3$ demultiplexer with central wavelength of $850 \mathrm{~nm}$. The three receiver channels do have peaks at 800,850 and $900 \mathrm{~nm}$, respectively. The average fiber-to-fiber insertion loss is $14 \mathrm{~dB}$ with a modal dependent loss of $<0.7 \mathrm{~dB}$ and a non-uniformity of $<2 \mathrm{~dB}$. The worst-case cross talk was found to be $-12.5 \mathrm{~dB}$.

The measured FSR corresponded to the value that was calculated based on the equations given in section II-C. For example, the FSR of the first output channel (at $800 \mathrm{~nm}$ ) was calculated to be $131 \mathrm{~nm}$ at the shorter wavelength side (corresponding to higher orders of the array). At the longer wavelength side (lower orders of the array) the FSR was calculated to equal $196 \mathrm{~nm}$. These values were experimentally verified.

\section{CONCLUSION}

A completely multimode $1 \times 3$ arrayed waveguide gratingbased demultiplexer has been demonstrated in the wavelength range that is used in LANs. The fiber-to-fiber insertion loss is $14 \mathrm{~dB}$ and the cross talk is $<-12.5 \mathrm{~dB}$.

The transmission peaks in the spectral response of the demultiplexer appear at the designed wavelengths. The FSR of the array could be accurately calculated based on the measured refractive indices of the materials.

The demultiplexer is compatible with multimode fiber and is fabricated using a low-cost polymer planar waveguide technology. A propagation loss of $0.5 \mathrm{~dB} / \mathrm{cm}$ and fiber-chip coupling loss of $1.3 \mathrm{~dB}$ in total have been obtained. The worst-case bend losses of a $10 \times 40 \mu^{2}$ waveguide were measured to be $0.4 \mathrm{~dB}$.

\section{ACKNOWLEDGMENTS}

The authors would like to thank the Dutch foundation for science and technology STW for financial support.

\section{REFERENCES}

[1] Brian E. Lemoff, Lewis B. Aronson, and Lisa A. Buckman, "SpectraLAN: A Low-Cost Multiwavelength Local Area Network," Hewlett-Puckard Journal, pp. 42-52, December 1997. 
[2] Meint K. Smit, Integrated Optics in Silicon-based Aluminum Oxide, PhD thesis, Delft University of Technology, Delft, The Netherlands, 1991.

[3] H. D. Bauer, W. Ehrfeld, M. Harder, T. Paatzsch, M. Popp, and I. Smaglinski. "Polymer waveguide devices with passive pigtailing: an application of LIGA technology," Synth. Met, vol. 115, pp. 13-20, 2000.

[4] Younan Xia, John A. Rogers, Kateri E. Paul, and George M. Whitesides, "Unconventional Mcthods for Fabricating and Patterning Nanostructures," Chem. Rev, vol. 99, pp. 1823-1848, 1999.

[5] H. Takahashi, K. Oda, H. Toba, and Y. Inouc, "Transmission Characteristics of Arrayed Waveguide $N \times N$ Wavelength Multiplexer," J. Lightwave Terhnol., vol. 13, pp. 447-455, March 1995.

[6] R. Adar, C.H. Henry, C. Dragone R.C. Kistler, and M.A. Milbrodt, "BroadBand Array Multiplexers Made with Silica Waveguides on Silicon," $J$ Lightwave Technol., vol. 11, pp. 212-219, February 1993.

[7] Microchem, hrtp://www.microchem.com, NANO SU-8 25.

[8] A. Borreman, S. Musa, A.A.M. Kok, M.B.J. Diemeer, and A. Driessen "Fabrication of Polymeric Multimode Waveguides and Devices in SU-8 Photoresist using Sclective Polymerization," Proc. IEEE/LEOS Symposium (Benelux Chapter), The Netherlands, December 2002.

[9] J. Rheims, J. Kose, and T. Wriedt, "Refractivc-index measurcments in the near-IR using an Abbe refractometer," Meas. Sci. Techn., vol. 8, pp. 601 $605,1997$.

[10] François Ladouceur, and John D. Love, Silica-based Buried Channel Waveguides and Devices, Chapman \& Hall, London, 1996.

[11] E.A.J. Marcatili, "Bends in Optical Dielectric Guides," Bell Sys. Tech. J, vol. 48, pp. 2103-2132, 1969. 